\title{
Acerca de la función de lo estético-cognitivo en el proceso de lecto-escritura para la adquisición de segundas lenguas
}

\section{María Isabel Barboza Ramírez}

Profesora de la Universidad de Costa Rica e Instituto Tecnológico. Investigadora en Adquisición de Segundas Lenguas. Poetiza.

Recibido: 9 de mayo de 2011

\section{RESUMEN}

Esta investigación gira en torno a que, mediante la respuesta del lector a textos literarios, desde un marco teórico bidireccional, estético-cognitivo, se puede observar la interacción y transacción entre el texto y el lector, a la vez que se coadyuva al proceso de enseñanzaaprendizaje de una segunda lengua. Dentro de la teoría cognitiva se tomaron como base los estudios seminales de Rumelhart, Anderson y Pearson, así como los estudios de Smith. En cuanto a la respuesta del lector sirvieron de fundamento la teoría de la Transacción Estética de Rosenblatt y los teóricos de la Recepción Estética: Culler, Jauss e Iser.

\section{PALABRAS CLAVE}

semiótica, adquisición de español como segunda lengua, literatura, lectura, interacción.
Aceptado: 19 de octubre de 2011

\begin{abstract}
SUMMARY
This investigation is based in the premise that from the reader's response to literary texts, studied from a bidirectional and esthetic-cognitive perspective, interaction and exchange between the text and the reader may be observed, and at the same time, this relation helps in the teaching-learning process of a second language. Seminal studies from Rumerhalt, Anderson and Pearson, and Smith were taken as basis inside the cognitive theory. For the reader's response, the Esthetic Transaction theory of Rosenblatt and the Esthetic Reception Theory from Culler, Jauss and Iser served as support.
\end{abstract}

\section{KEY WORDS}

Semiotics, Spanish as a second language acquisition, literature, reading skills, interaction.

El presente artículo plantea dos bases teóricas (lo estético-transaccional y lo esquemático-cognitivo) para la comprensión y lectura de textos literarios que permiten la interacción texto-lector en una forma dinámica y entretenida para la adquisición y aprendizaje de segundas lenguas. Lo novedoso de la función estética-cognitiva, se encuentra en la vigencia que todos estos autores dan a sus teorías, a la hora de ser aplicadas en una forma práctica, mediante el uso de textos literarios, a pesar de las fechas de publicación de sus artículos. El aporte se encuentra en la mezcla que como investigadora realicé de ambas teorías, para dar como resultado un acercamiento estratégico en la comprensión y lectura de una primera y segunda lengua por parte de los aprendices.

Asimismo, debo aclarar que la ML Marta Sánchez de la Universidad de Costa Rica inicia sus investigaciones en lengua inglesa desde este marco teórico dual, en 2001-2003. Más tarde yo retomo estos conceptos básicos en la adquisición del español como segunda lengua (2006). 


\section{- Antecedentes teóricos}

Los orígenes de la teoría del esquema se remontan al psicólogo francés Francis Barlett quien introdujo por primera vez el concepto de "esquema" en su libro "Remembering" Barlett (1932) pudo observar, a través de sus experimentos con sujetos lectores que ellos procesaban las historias leídas o escuchadas de forma diferente dependiendo de sus culturas. Él le dio a un grupo de lectores avanzados de diferentes culturas una versión arreglada de un cuento del folclore anglosajón. Los sujetos debían leer y luego escribir su evocación del cuento. Barlett observó significativas variaciones entre las distintas evocaciones y pensó que se debían a una organización y acomodamiento de la memoria, la cual él llamó "esquema". Barlett pensó que las diferencias entre "los esquemas" culturales de un individuo a otro eran los responsables de estas variaciones en la reproducción de las historias (en Sánchez, 1995). A partir de Barlett se siguió llamando "esquema" a las unidades de conocimiento responsables del acomodamiento de la información en la memoria. De ahí nace la teoría del esquema. Posteriormente, estudios seminales de Rumelhart (1977) retoman esta teoría, para dar paso a la función cognitiva y los procesos de acomodamiento de la información en nuestra mente.

Dentro del marco esquemático-cognitivo los estudios de Frank Smith (1988), Anderson y Pearson (1984) y David Rumelhart (1977), así como los planteamientos de luri Lotman (1998), desde la Semiótica de la Cultura ponen énfasis en el proceso de comprensión e interpretación (o distorsión) de un evento en la interacción texto-lector.

Frank Smith(1988) cuyas investigaciones giran especialmente en torno al aprendizaje de lectura en edades escolares, privilegia la "predicción" como elemento motor en la comprensión de lectura (para el aprendizaje en general.) En su libro Understanding Reading, Smith postula que la comprensión, en términos generales, se da como resultado de la "teoría del mundo en nuestra mente", resultante de las experiencias previas (esquemata) acumuladas en la memoria. El conocimiento previo o teoría del mundo en nuestro cerebro es el que establece la conexión con el contenido del papel impreso, de ahí que se dé "la predicción" en la comprensión de lectura.

Él explica:

What we have in our heads is a Theory of what the world is like, a theory that is the basis of all our perceptions and understanding of the world, the root of all learning, the sources of hopes and fear, motives and expectancies, reasoning and creativity.

Smith establece una relación vertical entre el cerebro y el entorno ("top down"). Considera que la dirección del aprendizaje reside en la capacidad de "predecir" a partir del conocimiento desde afuera, desde lo que nos rodea. La estructura cognitiva del cerebro contiene la disposición esquemática necesaria para el acomodo de la nueva información. Estos esquemas de conocimiento permiten explicar el proceso de lectura y la comprensión en general. Un ejemplo puede aclarar mejor este concepto. Si un individuo lee un texto sobre "béisbol" y posee los esquemas adecuados sobre el tema podrá tener una reacción predictiva y entenderá mejor la lectura que uno que no los tiene. Por eso, cuando Smith dice "La lectura depende de la predicción", se refiere a que el conocimiento previo permite la interpretación porque existen los esquemata que se activan a partir de ese mundo circundante. Desde el punto de vista de Smith, podría decirse que existe una capacidad "innata" del cerebro (es decir, que nace con el sujeto), a partir de los esquemata, para establecer asociaciones o paralelismos con el mundo exterior y así lidiar con situaciones inesperadas que pueden ser desde una palabra desconocida hasta un dato que el lector podría adivinar mediante "el contexto".

Igualmente, en relación con el elemento cognitivo, Rumelhart define los esquemas como 
grupos o paquetes dentro de los cuales está organizada la información en el cerebro. Él explica:

A schema is an abstract knowledge structure. A schema is abstract in the sense that it summarizes what is known about a variety of cases that differ in many particulars. An important theoretical puzzle

is to determine just how much and what sort of knowledge is abstracted and how much remains tied to knowledge of specific instances. (p.259)

Es decir, cada esquema puede comprender un campo enorme de conocimientos, actividades y situaciones porque constituyen modelos internos de conocimiento que son los que nos permiten la predicción.

En el contexto de la comprensión de lectura, Rumelhart explica la función del esquema de la siguiente forma:

Perhaps the central function of schema is the construction of an interpretation of an event, object, or situation - that is, in the process of comprehension...

The total set of schemata instantiated at a particular moment in time constitutes our internal model of the situation we face at that moment in time, or, in the case of reading a text, a model of the situation depicted by the text. (p.37)

Por ejemplo, si un lector tiene ante él un tratado sobre el proceso de conquista en América Latina y no dispone de los conocimientos mínimos sobre el tema, no podrá procesar el texto con la misma rapidez y efectividad con las que lo haría un lector poseedor de estos esquemas culturales. Pero el conocimiento previo se puede activar en los lectores con la ayuda del instructor y así acelerar el proceso de comprensión. En otras palabras, cuanto mayor conocimiento previo (esquemata) tenga un lector sobre el contenido de un texto, tanto mayor será su comprensión del material escrito, porque sus esquemas se verán activados conforme lee.

Rumelhart también ha dedicado sus investigaciones al estudio de la función de los esquemas de comprensión en la estructura narrativa, especialmente los cuentos por ser ésta la primera gramática que adquieren los niños. En torno a esto, Cuetos (1994) explica: "Rumelhart fue el primero que se interesó por la estructura de este tipo de narraciones. Comprobó que los cuentos están constituidos por categorías perfectamente separables, cada una de las cuales contiene un tipo de información específica. Distinguió dos categorías: la introducción y el episodio, en la primera se presentan los personajes principales y el contexto; la segunda está compuesta por un suceso y una reacción" (p.12). Sus investigaciones muestran cómo funcionan esos esquemas en edades tempranas puesto que los niños parecen anticiparse a la dinámica del cuento de hadas. Con este tipo de conocimiento previo se activan sus esquemas para la comprensión de lectura. En resumen, los esquemas, para Rumelhart son:

... a data structure for representing generic concepts stored in memory. There are schemata representing our knowledge about all concepts: those underlying objects, situations, events, sequence of events, actions and sequences of actions. (p.34)

Las investigaciones de Richard Anderson y David Pearson, en su artículo "A schema theoretic view of basic processes in reading comprehension" se enfocan en un aspecto: cómo los conocimientos previos o esquemata funcionan en la interpretación de la nueva información, de modo tal que ésta pueda organizarse y almacenarse en la memoria. Ellos prueban que el esquema puede ser activado con solo un detalle que se mencione. En relación con esta dinámica, consideran que la mayoría de las discusiones sobre la teoría de los esquemas enfatizan el papel de los esquemata para almacenar la información.

\section{- La interacción texto-lector en una segunda lengua}

Los estudios en torno a la adquisición y aprendizaje del lenguaje mediante la lectura en lengua materna, centran su interés en fundamentos teóricos que enfatizan la importancia 
de la interpretación y la respuesta del lector (Smith, Rosenblatt, Iser). Así, el proceso de la comprensión de lectura debe estudiarse desde la interacción de dos elementos: texto y lector, un constructo que depende de los esquemas que intervienen en el proceso. Todas las experiencias pasadas o conocimientos previos de lugares, situaciones, escenarios entre otros, que tenemos en nuestra mente, intervienen en la totalidad del proceso y forman la red esquemática que permite la predicción cuando leemos. La predicción es una apertura de nuestra mente hacia un potencial de proyecciones o alternativas que hace que el lector pueda crear nuevos mensajes interpretativos, a partir de los acomodamientos de insumos esquemáticos nuevos. Esto se ha de reflejar en la respuesta que, a su vez, se deriva de la o las interpretaciones del lector.

En el contexto de una segunda lengua, la teoría del esquema ha apoyado numerosos estudios en relación con los esquemata culturales, de contenido y de forma.

Elizabeth Bernhart (1991) en Reading Development in a Second Language, ofrece una valiosa base de datos de numerosos estudios en todas estas áreas. La recopilación comprende estudios entre 1970 y 1991. En opinión de Bernhart, el fenómeno de la comprensión lectora debe ser visto como un proceso cognitivo y social. Para la autora cada contexto cultural trae nuevas valoraciones al texto en la medida en que éste último esté abierto a múltiples interpretaciones. Dice lo siguiente:

When texts are viewed as cultural artifacts, they become fluid and open to multiple interpretations. In other words, they contrast the social view with the cognitive one above. The processing of text can be viewed only within a unique cultural context. Each cultural context will bring a different set of values into play (p.10).

Otro aspecto importante por considerar en la enseñanza de la comprensión de lectura es la motivación. En algunos casos en el proceso de lectura puede que no haya motivación porque el filtro afectivo del lector o del aprendiz de una segunda lengua es muy alto y existe un rechazo hacia la nueva cultura. No obstante, cuando se aprende una segunda lengua, por un deseo voluntario surge la necesidad de integrase a una sociedad o grupo social y el filtro afectivo es menor. En este caso hay una motivación para ser aceptado puesto que existe "un sistema de valores que se debe compartir en una segunda cultura" (Bernhardt, 1991: 21). Esa experiencia cultural será mejor o peor según el grado de aceptación y de conocimiento del nuevo código lingüístico y cultural. Por esta razón, la literatura es tan relevante. Ningún texto literario es ajeno a la cultura que rodea al autor. En este sentido, la familiaridad cultural entre el texto y el lector permitirá un mayor acercamiento entre ambos. Este acercamiento es el que habría que inducir en algunos casos, puesto que no todos los lectores comparten los mismos esquemas antes mencionados. Lugares, personajes, escenarios forman una cadena infinita de evocaciones para el lector. De esa cadena puede desprenderse una gran variedad de respuestas ya que cada individuo percibe y procesa la información de manera distinta. De hecho, el foco de atención varía de un individuo a otro, y por ende, el proceso de comprensión y de transacción estética también.

Varios elementos intervienen en este proceso como la sintaxis, las configuraciones de ideas entre otros, que forman parte de lo que tanto Smith como Rosenblatt llaman "convenciones de la lengua". Al respecto Frank Smith dice: "Todos los aspectos del lenguaje son convencionales, empezando desde los muchos sonidos y significados de las palabras que usamos" (44, Mi traducción). Smith coincide con la mayoría de los expertos en que estas convenciones en una lengua son representaciones y significados arbitrarios que cambian de una lengua a otra y que son bases esenciales para la comprensión y comunicación.

En esta misma dirección luri Lotman (1998) dice:

El pensamiento es un acto de intercambio, y por consiguiente presupone una actividad bilateral. El texto introducido desde el exterior, estimula, "conecta" la 
conciencia. Pero para que esta "conexión" se produz$c a$, el dispositivo que es conectado debe tener fijada en su memoria una experiencia semiótica, es decir, ese acto no puede ser el primero... Podemos distinguir, como mínimo, tres clases de objetos inteligentes: la conciencia natural del hombre (de una unidad humana aislada), el texto (en la segunda aceptación) y la cultura como inteligencia colectiva." (p.17)

En otras palabras, tanto las imágenes "verbales" como "no verbales" (íconos, ilustraciones) en un sistema lingüístico dado, pueden ser determinantes en el proceso de la comprensión de lectura y en la respuesta del lector. Estas imágenes, activan un sistema esquemático que está ligado a la memoria colectiva. En este sentido podemos entender a Rumelhart cuando dice que la teoría del esquema "encarna el prototipo de una teoría del significado en la medida en que un esquema subyacente a un concepto almacenado en la memoria corresponde al significado de ese concepto" (257). Así, los significados son decodificados en términos de las situaciones o eventos típicos o normales que se acomoden a dicho concepto.

En realidad, ninguna imagen puede ser entendida si previamente no está asociada a lo que Lotman llama "un dispositivo pensante", es decir, algo que conecta al cerebro humano a una imagen anterior o previa. Estas imágenes para Rumelhart estarían contenidas en los esquemas o "piezas constructoras de la cognición", si estas piezas no existen sería difícil que se dé esta conexión de la que habla Lotman. En el caso de Smith, son una condición sine qua non de "el corazón del proceso de lectura", por lo tanto, permiten la predicción.

Lo anterior es especialmente válido dentro del campo de la comprensión de lectura de una $L 2$, ya que, existen lectores cuyo sistema de representación iconográfico de su primera lengua, la escritura por ejemplo, es totalmente distinto al de la lengua meta. Tal es el caso de los estudiantes orientales (chinos, japoneses, coreanos, tailandeses, etc.), cuyos esquemas lingüísticos y culturales están más alejados de los hispanoparlantes. Smith señala que el sistema alfabético en una lengua es más fácil de aprender a escribir que de leer. Por ejemplo, hablantes de inglés y francés no pueden entenderse a menos que ellos estén usando una pequeña parte de su sistema de escritura que no es alfabético, como símbolos aritméticos $2+3=5$.

Por eso toda experiencia de aprendizaje de una segunda lengua puede constituir una experiencia cultural y lingüística total, en la que se involucran esquemas nuevos de contenido y forma del texto, incluyendo desde las estructuras culturales y lingüísticas, hasta el sistema representativo o iconográfico tanto de la cultura como de la lengua.

\section{- La respuesta del lector}

En relación con los teóricos de la Respuesta del Lector, Wolfgang Iser, Hans Robert Jauss, CuIler (1987) y Rosenblatt (1978) plantean la lectura del texto literario como un acto estético, es decir, de respuesta a los efectos o evocaciones que el texto literario produce en el lector durante el proceso de comprensión.

Desde la teoría de la transacción estética L.M Rosenblatt ofrece un marco metodológico y conceptual que permite abordar los estudios literarios y el proceso de la comprensión lectora al mismo tiempo, dentro del campo de la enseñanza de la literatura. La lectura transaccional no es una reproducción de la información sino una experiencia lúdica provocada por el texto. No obstante, para que esto ocurra, debe existir un "ambiente" propicio para el lector tal como: el estado de ánimo, momento de la vida entre otros; a lo que Rosenblatt denomina el surgimiento del "poema", es decir el momento de creatividad.

En cuanto a la teoría de la Estética de la Recepción, Wolgang Iser (1987) propone que:

En las obras literarias ocurre una interacción en cuyo transcurso el lector 'recibe' el sentido del texto en el 
proceso en que él mismo lo constituye. En lugar de

la existencia previa de un código determinado, en cuanto al contenido, surgiría un código en el proceso de constitución, en cuyo transcurso la recepción del mensaje coincidiría con el sentido de la obra..(p.123)

Es decir, a partir del lector se crea el mensaje, no existe un código preestablecido, lo cual le permite una multiplicidad de interpretaciones de acuerdo con sus experiencias vividas. A la hora de comprender un texto, puesto que ningún texto es unilateralmente determinante, es decir, en términos de la interpretación, el lector eficiente trasciende lo meramente impreso y llena los gaps que persisten de los esquemas activados, también llamados por Iser, "los blancos o espacios vacíos". Estos gaps los llena el lector al relacionar los segmentos textuales que estimulan el proceso comunicativo entre texto-lector.

Desde el punto de vista de la teoría de la Respuesta del Lector, algunos teóricos han utilizado textos literarios para el aprendizaje de inglés como L2. Por ejemplo, James Davis (1989) se basa en los planteamientos de Wolfgang Iser, teórico de la recepción, y muestra que los textos literarios permiten una mayor interacción texto-lector porque reactivan la creatividad en este último, viéndose esta reflejada incluso en la respuesta escrita. La respuesta se enriquece en la medida en que el texto evoque en el lector experiencias personales y culturales. Davis (1989) concluye:

The literary text is considered as an efficient vehicle for foreign language acquisition, as an organic whole for cultural analysis, and as a non-banal context for composition writing. (p.20)

El autor se basa en que, por su naturaleza, el texto literario ofrece elementos contextuales -históricos, culturales-, los cuales, junto con otros componentes reconstructivos y creativos que provienen tanto del autor como del lector, enriquecen el proceso.

Wolfgan Iser (1987) en su artículo "El acto de la lectura" nos remite a las posibilidades del lector como partícipe, como ente activo en el proceso de comprensión lectora. Él dice:

La obra literaria posee dos polos que se podrían denominar el polo artístico y el polo estético, el polo artístico designa al texto creado por el autor y el polo estético designa la concretización efectuada por el lector... Alli pues, donde el texto y el lector convergen, se halla el lugar de la obra literaria y éste tiene forzosamente un carácter virtual, ya que no puede ser reducido ni a la realidad del texto, ni a las predisposiciones que caracterizan al lector. De esta virtualidad de la obra nace su dinámica la que a su vez forma la condición para el efecto provocado por la obra. El texto alcanza, por consiguiente, su existencia a través del trabajo de constitución de una conciencia que lo recibe, de manera tal que la obra puede desarrollarse hasta su verdadero carácter como proceso sólo en el curso de la lectura. Por eso, de aquí en adelante, sólo se deberá hablar de una obra cuando este proceso se realice dentro del procedimiento de constitución reclamado por el lector y producido por el texto. La obra es el hecho-consti-

tuido del texto en la conciencia del lector. (p.2)

Asimismo, Jauss (1987) opina:

La experiencia estética no comienza con el reconocimiento y la interpretación del significado de una obra, ni mucho menos con la reconstrucción de la intención de su autor. La experiencia primaria de una obra de arte se realiza en la actitud respecto a su efecto estético, en la comprensión que goza y en el goce comprensivo. (p.75)

Por lo tanto una respuesta lectora debe provenir no solo de la comprensión sino del placer estético del acto de lectura, como un acto de "efecto y recepción".

\section{Culler (1987) sostiene que:}

Lectores diferentes pueden descubrir diferentes formas de unidad, pero las formas básicas de sentido que buscan pueden ser las mismas.(p.144)

Ahora bien, si la lectura de un texto literario es una experiencia estética tal y como lo plantean los autores citados, ésta ocasionará de hecho una transacción, una negociación entre el 
texto y el lector. Por lo tanto el acto de lectura no está en la intención del autor ni en el "posible significado" de la obra, sino en la respuesta estética del lector. Sin duda distintos lectores generarán múltiples interpretaciones a partir de la relación e interacción texto-lector. Culler (1987) plantea que:

Una teoría de la lectura tiene que poner de relieve las operaciones interpretativas utilizadas por los lectores. Todos sabemos que diferentes lectores producen diferentes interpretaciones. (p.44)

Desde el punto de vista de la teoría de la Respuesta del Lector, tanto Rosenblatt (1978) como los teóricos de la Recepción Estética, Iser, Jauss, y Culler (1987), el texto cobra vida a partir de la lectura que deviene de un potencial lector, es decir, de las posibilidades de reacción de ese lector mediante una respuesta estética. Por lo tanto, constituye "un constructo orgánico vital" (Rosenblatt, 1978) en la medida en que se crean los significados a partir de esa respuesta personal del lector.

Cuando el lector entra a participar de la experiencia estético-cognitiva es de hecho un ser social, cultural e histórico, con lo cual aporta esas nuevas valoraciones al texto. Por otra parte, también es un lector imaginativo. Este último, crea e imagina dentro de la perspectiva estético- cognitiva (Rosenblatt, 1978; Sánchez, 2001).

Rosenblatt, teórica de la respuesta estética en el contexto de la comprensión de lectura en la lengua materna, añade una observación pertinente también a la comprensión de lectura en una segunda lengua. Ella dice: "Como con todos los textos el lector debe aportar más que la comprensión literal de cada palabra. Debe aportar un cuerpo completo de supuestos culturales, conocimiento práctico, conciencia de las convenciones literarias, una apertura para pensar y sentir" (p.12). De acuerdo con la autora, en el acto de lectura, el lector trae al acto todo su bagaje de significantes culturales, lingüisticos y personales, incluyendo su disposición emotiva.
Rosenblatt ha publicado varios libros y artículos alrededor de sus propias experiencias como profesora de literatura inglesa y a partir de estas experiencias, deja las bases para la investigación empírica en la comprensión de lectura más allá del concepto cognitivo tradicional. Para la autora, la transacción estética es un constructo orgánico en el que ambos, texto y lector "se justifican" mutuamente, es decir, tanto el lector como el texto crean el "poema" en el proceso de interpretación (Sánchez, 2001).

El texto literario es un constructo de símbolos lingüísticos que pueden activar en el lector imágenes sensoriales que éste asocia con sus experiencias de vida. Rosenblatt en su libro, The reader, the text, the poem dice:

The reader's attention to the text activates certain elements in his past experience- external reference, internal response - that have become linked with the verbal symbol. Meaning will emerge from a network of relationships among the things symbolized as he senses them. The symbols point to these sensations, images, objects, ideas, relationships, with the particular association or feeling-tones created by his past experiences with them in actual life

or in literature. (p.11)

Peter y Julie Prest (1988) basados en la aplicación del método transaccional de Rosenblatt en sus clases de literatura inglesa para escolares, muestran que la respuesta del lector lleva a sorpresivos resultados en la comprensión lectora de textos literarios, en edades tempranas. Ambos señalan que algunas respuestas fueron emanadas propiamente de la información textual y otras privilegiaron lo personal-vital, procesos que equivalen a lo eferencial y a lo estético respectivamente. (Rosenblatt, 1978)

Ellos dicen en relación con el primer aspecto:

Their transactions with the text will be made in terms of materials taken away, details remembered, ideas gleaned to be applied to some further endprojects, essays, exams. It is primarily this efferent mode which allows students to map out their schema of literary awareness, schema which becomes useful for them when they need to identify, locate, 
and predict the nature and form of the material in front of them. (p.130)

En relación con el segundo aspecto Meek (1983) afirma que, "los lectores satisfechos son por definición lectores estéticos y quizá sus mejores maestros pudieron ser los autores de los libros que ellos disfrutan" (p.14). Y por su parte, Prest sostiene que cualquier actividad que promueva la respuesta estética debe permitir a los estudiantes permanecer como socios activos en la relación texto-lector.

Igualmente basada en el modelo transaccional, y refiriéndose a la respuesta escrita, Norma A. Greco (1991), en su artículo "Re-creating the literary text" dice lo siguiente:

I have found that one of the most effective ways in which teachers can help their students become "producers" and in so doing transform the reading and study of literature into more creative and meaningful experience is through certain focused writing assignments that challenge students to become agents in the construction of textual meaning. (p.34)

Para la autora, la lectura tradicional de textos literarios ha convertido a los estudiantes en simples "consumidores". A través de sus experiencias ella ha podido observar que el proceso de lecto-escritura, por ejemplo, lleva a beneficiosos resultados que muestran que el lector pasa de "consumidor" a "productor" o a cocreador del texto.

Igualmente Priscila L. Griffith y Katryn Lamframboise (1998), comprueban que las incursiones en la literatura como óptica de respuesta del lector generan reacciones tanto cognitivas como afectivas. Ellas realizaron estudios con adultos y niños utilizando la técnica de casos, considerando estos dentro de las historias, y observaron en sus resultados valiosos beneficios didácticos incluyendo la apertura a grupos de discusión dentro de sus clases. Ellas plantean que el método de casos permite al profesor recuperar los principios enseñados y aplicarlos en las situaciones de la clase a través de una discusión que enfatice la reflexión y resuelva problemas.

Esta realidad se hace más compleja, desde luego, en el proceso de enseñanza-aprendizaje de una segunda lengua. Por lo tanto, lel instructor debe considerar todos estos componentes dentro de las múltiples estrategias de enseñanza de la literatura. De esta manera la segunda lengua se convierte en una lengua viva que puede fluir en el lector para que éste, motivado por el texto, produzca nuevos mensajes.

En resumen, el texto literario ofrece una gama de posibilidades de respuesta. En el caso del lector de una segunda lengua, la transacción se abre a una multiplicidad de negociaciones con la lectura que involucra esquemas culturales y lingüísticos. En este sentido, Rosenblatt, Culler, Iser y Jauss comparten un denominador conceptual común. Todos ellos consideran que el encuentro entre el texto literario y el lector permite muchas interacciones desde una perspectiva estética garantizada por la misma permeabilidad del texto. El lector lee y responde, en forma oral o en forma escrita. Por eso, dentro de las posibilidades en el aula, en el ámbito de la enseñanza de una segunda lengua, se da un mayor intercambio cultural, discusiones e interacción entre los miembros, proceso interactivo y transaccional que enriquecerá las posibilidades didácticas y el proceso adquisitivo de la lengua.

Como investigadora en el campo de la función de lo estético-cognitivo en el proceso de lectoescritura en una primera y segunda lengua, debo agregar que en la etapa inicial de la investigación, trabajé con 12 lectores aprendices del español como segunda lengua en la Universidad de Costa Rica, todos ellos de diferentes nacionalidades (Japón, Italia, Alemania, Suiza, Estados Unidos, Noruega y Taiwan. En una segunda instancia, trabajé con 14 estudiantes de los Estados Unidos que tomaban el curso de Literatura Latinomericana y alrededor de 60 estudiantes del curso de Redacción Oral y Escrita de la Universidad Nacional de Costa Rica. 
Y finalmente con estudiantes de posgrado de la carrera de francés de esta misma universidad. Luego de aplicar ambas teorías tanto desde la transacción estética como desde la teoría del esquema en una primera y segunda lengua, sin duda, éstas ayudan a lograr grandes insumos en la enseñanza e investigación de la comprensión de lectura mediante el uso de textos literarios. En lengua materna se logra que los estudiantes estructuren frases más claras y por ende comprensibles, desde la frase tópica hasta las secundarias, así como les permite redactar párrafos descriptivos y narrativos en donde se requiere la creatividad, con lo cual se logra un mejor manejo del lenguaje. Por otro lado, la lectura/ escritura que envuelve el proceso propicia mayor habilidad en la elaboración de un documento o escrito; así como estimula todos los otros mecanismos sintácticos y léxicos que presupone la redacción de un documento. En cuanto a la enseñanza de una segunda lengua, la interacción /transacción permite mover todas las otras destrezas de la lengua: producción, expresión, comprensión, escucha y por supuesto, las estructuras gramaticales. Todo el proceso se da en forma natural y entretenida, de manera que los estudiantes en general disfrutan del acto de lectura, tal y como lo he comprobado a través de mi experiencia como docente.

\section{NOTAS}

1. Lo que tenemos en nuestra mente es una teoría de cómo es el mundo, una teoría que es la base de todas nuestras percepciones y el entendimiento del mundo, la raíz de todo aprendizaje, las fuentes de las esperanzas y el temor, móviles y expectativas, razonamiento y creatividad ( $7, \mathrm{Mi}$ traducción )

2. Un esquema es una estructura abstracta de conocimiento. Un esquema es abstracto en el sentido de que resume lo que se sabe sobre una variedad de casos que se diferencian en muchos detalles. Un importante rompecabezas teórico es determinar cuánto y qué clase de conocimiento es abstracto y cuánto se mantiene enlazado al conocimiento de las instancias específicas. ( 259, Mi traducción )

3. Tal vez la función central del esquema es la construcción de una interpretación de un evento, objeto o situación --o sea, en el proceso de la comprensión... La serie total de los esquemata activados en un momento particular constituye nuestro modelo interno de la situación que afrontamos en ese momento en el tiempo, o en el caso de la lectura de un texto, un modelo de la situación descrita en el texto (37, Mi traducción)

4. Un esquema entonces es una estructura de datos para representar los conceptos genéricos almacenados en la memoria. Hay esquemata que representa nuestro conocimiento sobre todos los conceptos: aquellos objetos subyacentes, situaciones, eventos, secuencia de eventos, acciones y secuencia de acciones (34, Mi traducción).

5. Cuando los textos se perciben como artefactos culturales se tornan fluidos y abiertos a múltiples interpretaciones. En otras palabras contrastan la visión social con la cognitiva. El procesamiento del texto puede verse sólo dentro de un contexto cultural único. Cada contexto cultural aportará una serie de diferentes valores a la interacción. (10, Mi traducción)

6. Un texto literario es considerado un vehículo eficiente para la adquisición de una lengua extranjera, como un todo orgánico para el análisis cultural, como un contexto nada banal para la composición (420, Mi traducción)

7. La atención del lector hacia el texto activa ciertos elementos de su experiencia pasada - referencia externa, respuesta internaque se han enlazado al símbolo verbal. El significado emergerá de una red de relaciones entre las cosas simbolizadas tal como las percibe. Los símbolos apuntan a estas sensaciones, imágenes, objetos, ideas, relaciones con esta asociación particular o tonos emocionales creados por sus experiencias pasadas en su vida actual o en la literatura (11, Mi traducción) 
8. Sus transacciones con el texto serán en términos de los materiales extraídos, los detalles recordados, ideas seleccionadas para aplicarse a proyectos futuros, ensayos, exámenes. Principalmente este modo de eferencia permite a los estudiantes delinear un esquema de conocimiento literario. Un esquema que se torna útil para ellos, cuando necesitan identificar, localizar y predecir la naturaleza y forma del material que tienen frente a ellos (130, Mi traducción)

9. He encontrado que una de las formas más efectivas para que los docentes puedan ayudar a sus estudiantes a convertirse en " productores" y así transformar la lectura y el estudio de la literatura en una experiencia más creativa y significativa, es a través de ejercicios de escritura enofocados a retar a los estudiantes a convertirse en agentes de construcción del significado textual (34, Mi traducción).

\section{BIBLIOGRAFÍA}

Anderson, R \& Pearson.(1984). D. A schema-theoretic view of basic processes in reading comprehension. In Pearson P.D. (Ed.), Handbook of Reading Research, Nueva YorK, 255-81.

Barlett, C.(1932). Remembering .The Cambridge University Press.

Bernhart, E.(1991) Reading Development in a second language. New Jersey: Alex Publishing Corporation.

Culler, Jonattan.(1982) Sobre la deconstrucción. Teoría y Crítica después del estructuralismo, Madrid, Cátedra.

Culler, Jonattan. (1979). La poética estructuralista, la lingüística y el estudio de la literatura, trad. Carlos Manzano, Barcelona.

Cuetos, Fernando.(1994). Psicología de la lectura. Madrid: Editorial Escuela Española, 23-51.
Dietrich, Rall.(1987).En busca del texto. Teoría de la Recepción Literaria. México: Editorial Universidad Autónoma de México, 121-143, 73-87.

Greco, N. (1991). Re-creating the literary text: Practice and Theory. English Journal, 85, 334-40.

Iser W. (1987). El acto de la lectura. En: Dietrich, Rall. En busca del texto. Teoría de la Recepción Literaria. México: Editorial Universidad Autónoma de México, 121-143.

Iser W, (1987). La interacción texto-lector. En: Dietrich, Rall. En busca del texto. Teoría de la Recepción Literaria. México: Editorial Universidad Autónoma de México, 351-364.

Jauss, H.R (1987). Horizonte de Expectativas. En: Dietrich, Rall. En busca del texto. Teoría de la Recepción Literaria. México: Editorial Universidad Autónoma de México,73-87

Jauss, H.R (1987). Historia de la literatura como una provocación a la ciencia literaria. En: Dietrich, Rall. En busca del texto. Teoría de la Recepción Literaria. México: Editorial Universidad Autónoma de México, 53-61

Jauss, H.R.(1977). Experiencia estética y hermenéutica literaria, Taurus, Madrid.

Lotman, Iuri.(1998). La Semiosfera. Semiótica de la Cultura del texto, de la Conducta y del Espacio. Madrid: Fronesis Cátedra Universitad de Valencia, 11-25.

Lotman, luri y Escuela de Tartu (1979). Semiótica de la Cultura. Madrid: Editorial Cátedra.

Prest. P \& Prest. J.(1988). Theory Practice. Clarifying our intentions: Some Thoughts on the theory of reading in the classroom. English Quartely, $21,2,127-33$.

Raman, Selden.(1998). La Teoría Literaria Contemporánea. Barcelona: Editorial Ariel, 127-146.

Rosi- Landi, Ferrucio. Semiótica y Estética. Buenos Aires: Ediciones Nueva Visión, 1972.

Rosenblatt, L.(1985).Viewpoints: Transacción versus Interacción-A terminological Rescue Operation. Reserch in the Teacher of English, 19, 1, 96107,1985 
Rosenblatt, L. M. (1978). The Reader, the Text, the Poem. Illinois. Soutern Ilinois University Press.

Rumelhart, D.(1977). Schemata: The building blocks of cognition. In Spiro, R.J et al. (Eds), Theorical Issues in Reading Comprehension. New Jersey: Lawrence Erlbaum, Associates. New Jersey.

Sánchez, Marta.(2001). El uso de textos literarios en la enseñanza de la composición en una lengua extranjera: una propuesta metodológica respaldada por la teoría el esquema y la teoría de la transacción estética de L.M.Rosenbatt. Repertorio Americano. Costa Rica, 12, 135-147.

Smith, F.(1988) Understanding Reading. New Jersey: Lawrence Erlbaum, Associate Publishers. 